\title{
Effect of flecainide on atrial fibrillatory rate in a large animal model with induced atrial fibrillation
}

Eva Z. Hesselkilde ${ }^{1}$, Helena Carstensen ${ }^{1}$, Maria M. Haugaard ${ }^{1}$, Jonas Carlson², Steen Pehrson ${ }^{3}$, Thomas Jespersen ${ }^{4}$, Rikke Buhl ${ }^{1 *}$ (D) and Pyotr G. Platonov ${ }^{5}$

\begin{abstract}
Background: Atrial fibrillatory cycle length has been considered one of the indices of atrial electrical remodelling during atrial fibrillation (AF), which can be assessed from surface ECG by computer-assisted calculation of atrial fibrillatory rate (AFR). Horses have been suggested as a bona fide model for AF studies since horses too, develop lone AF, however data on AF characteristics in horses are extremely sparse and non-invasive characterization of AF complexity using surface ECG processing has not been reported.
\end{abstract}

Aim: The aim was to study characteristics of induced AF and its modification by flecainide.

Methods: The study group consisted on 3 horses with spontaneous persistent AF and 13 with pace-induced AF. Seven horses were treated with saline (control) and eight with flecainide (2 mg/kg). ECGs were analysed using spatiotemporal cancellation of QRST complexes and calculation of AFR from the residual atrial signal.

Results: At AF onset, AFR was $295 \pm 52$ fibrillations per minute (fpm) in the horses with induced AF treated with flecainide, $269 \pm 36 \mathrm{fpm}$ in the control group (ns), and $364 \pm 26 \mathrm{fpm}$ in the horses with spontaneous persistent AF $(P<0.05$ compared to the control group). Flecainide caused a decrease in AFR in all animals and restored sinus rhythm in the animals with induced AF. In the control animals, AFR increased from $269 \pm 36 \mathrm{fpm}$ to a plateau of $313 \pm 14 \mathrm{fpm}$ before decreasing to $288 \pm 28 \mathrm{fpm}$ during the last $10 \%$ of the AF episodes preceding spontaneous conversion $(P<0.05)$.

Conclusion: AFR in horses with induced AF resembles AFR in humans with paroxysmal AF. Flecainide caused a rapid decrease in AFR in all horses, further supporting the method to be a non-invasive technique to study the effect of antiarrhythmic compounds.

Keywords: Antiarrhythmic drug, Atrial electrophysiology, Atrial fibrillation, Atrial fibrillatory rate, Animal model, Equine, Flecainide, Horse, Programmed electrical stimulation

\section{Background}

Atrial fibrillation (AF) is a self-sustained arrhythmia that progresses over time as a consequence of atrial remodelling. Shortening of the atrial refractory period is considered to be the hallmark of the electrical remodelling, and is closely linked to the atrial fibrillatory cycle length or its reversed measure atrial fibrillatory rate (AFR)

\footnotetext{
*Correspondence: rib@sund.ku.dk

${ }^{1}$ Department of Veterinary Clinical Sciences, Faculty of Health and Medical Sciences, University of Copenhagen, Højbakkegaard Allé 5, 2630 Taastrup, Denmark

Full list of author information is available at the end of the article
}

expressed in fibrillations per minute (fpm) [1]. As a noninvasive measurement of atrial remodelling, AFR has been repeatedly validated against intracardiac recordings $[1,2]$ and is expected to become a useful tool for assessment of AF treatment strategies.

Recently, the horse has been proposed as a bona fide model for AF. [3, 4] Like humans, horses develop AF both with and without underlying cardiac diseases, which is believed to be a consequence of their large hearts and high vagal tone. [5] In addition to the naturally occurring cases, AF can be electrically induced in horses, allowing studies of AF remodelling and treatment regimes. 
In clinical medicine, flecainide is a commonly used drug to restore sinus rhythm in patients with recentonset AF. [6] There is an ongoing debate over the effectiveness and safety of flecainide as an antiarrhythmic drug in horses, as the literature shows conflicting results. A study by Ohmura et al. [7] describes flecainide to be safe and efficient in restoring sinus rhythm in an experimental setting, whereas a Belgian group reported disappointing efficacy [8] and fatal side effects. [9] AFR has been reported to decrease after flecainide treatment [3], but whether non-invasive AFR assessment is suitable for monitoring drug effects in horses (based on the processing of surface electrocardiogram (ECG) recordings) is yet to be studied.

\section{Methods}

The aim of this study was to assess AFR behaviour in horses with atrial fibrillation and to study the effects of flecainide on AFR using time-frequency analysis applied to Holter ECGs.

ECGs from 13 Standardbred horses were collected. All horses were electrically stimulated into AF using programmed electrical stimulation from a multipolar catheter placed in the right atrium through the jugular vein. After AF induction the horses were either treated with saline (control group) or flecainide (flecainide group). Continuous modified base-apex ECG was recorded during the entire duration of the experiments. [3] The control group consisted of four mares and three geldings (mean age 9.1 years, range 4-14; mean bodyweight (BW) $497 \mathrm{~kg}$, range 454-555). The flecainide group consisted of five mares and two geldings (mean age 7.4 years, range 4-16; mean BW $474 \mathrm{~kg}$, range 390-552). One horse received both saline and flecainide with a 6-day wash-out period in between.

Following AF induction, a minimum of 15 min continuous AF was required before initiation of treatment with either Flecainide (Tambocor, Meda AS, Allerød, DK; $n=7)$ or isotonic saline $(\mathrm{n}=7)$. Flecainide $(2 \mathrm{mg} / \mathrm{kg})$ or saline were infused intravenously (IV) over a period of $10 \mathrm{~min}$. Five horses received three additional IV saline injections $(0.0125 \mathrm{ml} / \mathrm{kg} / \mathrm{min}) 20 \mathrm{~min}$ apart. After infusion the horses were monitored at rest until either drug induced or spontaneous cardioversion occurred. In order to minimize the risk of untimely spontaneous cardioversion in horses scheduled for saline infusion, they were required to have an $\mathrm{AF}$ episode of at least 1-h duration to be included in the study.

Additionally, three horses with spontaneous AF documented with long-term continuous ECG registration were included in the study (two Standardbreds, one Warmblood), one mare and two geldings (mean age 8 years, range 7-10; mean BW 514 kg, range 464-548).
Horse A had successfully been treated for AF with Quinidine Sulfate twice within recent years. The AF episode included in this paper had lasted more than 3 weeks prior to flecainide treatment as described above. The AF duration in horse B was also known to be over 3 weeks, whereas it was only possible to determine that the AF duration in horse $\mathrm{C}$ was a minimum of $12 \mathrm{~h}$, as AF was an incidental finding and the horse was brought straight to the clinic. There were no records of previous AF episodes for horse $\mathrm{B}$ or $\mathrm{C}$ and no flecainide treatment were attempted.

\section{ECG recordings and signal processing}

ECGs were recorded as a modified base-apex ECG. All recordings for the induced horses included AF onset, saline or flecainide infusion and termination of AF, while the spontaneous persistent horses had minimum of $60 \mathrm{~min}$ ECG recorded and analysed. All ECG recordings were manually reviewed for quality assessment and the presence of AF in the Televet system (Kruuse, Langeskov, DK) or Labchart software (LabChart 7 Pro, ADInstruments, Oxford, UK). ECG analysis including QRST cancellation and AFR calculation was performed using the Cardiolund AFR Tracker software (cardiolund.com). [10] AFR values where less than $10 \%$ of the signal could be used for automated analysis were discarded as missing values.

\section{Statistical analysis}

Data are presented as mean \pm SD or mean \pm SEM as appropriate. To account for different AF duration when studying AFR behaviour in the control horses, the median of the AFR values in the 10th, 45-55th and 90100th percentiles of AF duration in the individual horses were calculated, and a Friedman's one-way ANOVA was performed, followed by Dunnett's post hoc test. AFR values were compared between groups using a MannWhitney unpaired t-test, while a comparison of AFR values before and after treatment was performed using Wilcoxon matched pairs t-test where the mean of the last 10 min prior to treatment was compared to the last AFR value before cardioversion (flecainide group) or mean of the following $10 \mathrm{~min}$ after injection start (control group). The threshold for statistical significance was $P<0.05$ throughout the study.

\section{Results}

The quality of the ECG recordings was generally good and few segments (5.3\%) of the ECGs were discarded due to excessive levels of noise.

For the AF-induced horses, the mean AF duration in the control group was 205 min (range 152-273) and 26 min (range 16-36) in the flecainide group. 
Table 1 AFR behaviour in the control horses

\begin{tabular}{lll}
\hline & AFR (fpm) & Time (minutes) \\
\hline Initiation of AF & $269 \pm 36^{\dagger}$ & 1 \\
0-10th percentile & $299 \pm 10^{\dagger}$ & $21 \pm 4.9$ \\
45-55th percentile & $313 \pm 14$ & $92 \pm 22-112 \pm 27$ \\
90-100th percentile & $302 \pm 19^{\dagger}$ & $183 \pm 44-204 \pm 49$ \\
Last value prior to termination & $288 \pm 28^{\dagger}$ & $205 \pm 50$ \\
\hline
\end{tabular}

AFR (fpm) and time (minutes) described at initiation of AF, the first, middle and last $10 \%$ of the AF episodes, and the last value before termination. All values are presented as mean \pm SD

${ }^{\dagger}$ Differs significantly from the $45-55$ th percentiles $(P<0.05)$

\section{AFR dynamics in control horses during induced AF}

AFR at initiation of AF was $269 \pm 36$ fpm (range 208317). AFR significantly increased in all horses from the 10 th percentile $(299 \pm 10 \mathrm{fpm})$ to the $45-55$ th percentile $(313 \pm 14 \mathrm{fpm} ; P<0.05)$. Before spontaneous cardioversion, AFR significantly decreased again at the 90-100th percentile $(302 \pm 19 \mathrm{fpm} ; \mathrm{P}<0.05)$. Table 1 describes the AFR and time at the different stages. The difference between percentiles suggests a plateau or "steady state" during which AFR was stable. Studying the individual trends, the time to the plateau was 40 to $70 \mathrm{~min}$ in six of the horses, while AFR in one horse continued to increase for $140 \mathrm{~min}$. The average time to plateau was 68 $\pm 33 \mathrm{~min}$, as illustrated in Fig. 1. All horses showed a decrease in AFR during the last $30 \pm 17 \mathrm{~min}$ prior to spontaneous cardioversion.

\section{The effect of flecainide on AFR during induced AF}

At AF onset, AFR was $295 \pm 52 \mathrm{fpm}$ in the flecainide group, which was non-significant compared to the control group $(P>0.05)$. Flecainide restored sinus rhythm in all horses with induced AF. The duration of AF episodes before treatment was on average $22 \pm 5 \mathrm{~min}$, and time to cardioversion after flecainide infusion started was $4.3 \pm$ $2.5 \mathrm{~min}$. AFR decreased immediately after the initiation of flecainide infusion $(P<0.05)$. In contrast, AFR in the control group remained unchanged after saline infusion $(P>0.05)$. Figure 2 illustrates the behaviour of AFR after infusion with flecainide (2a) and saline (2b).

\section{AFR in horses with spontaneous persistent AF}

The horses with spontaneous persistent AF presented with a significantly higher AFR compared to the horses with electrically induced AF $(P<0.05$; mean of the first AFR value recorded $364 \pm 26$ ) and remained stable in the absence of drug intervention (Fig. 3a). Sinus rhythm was not restored in response to flecainide administration in horse A, despite a decrease in AFR of more than $100 \mathrm{fpm}$ 10 min after infusion (Fig. 3b).

\section{Discussion}

\section{Main findings}

In the present study, we found that AFR in horses closely resembles AFR in humans, both in terms of rate, behaviour over time, and response to drug injection. We found an AFR range of 250-350 fpm in seven horses with induced AF, which is well correlated with clinical observations where patients with induced AF had a frequency of $336 \pm 78$ beats/min, [2] and patients with paroxysmal AF reached a rate of $326 \pm 39 \mathrm{fpm} 3 \mathrm{~h}$ after onset. [11] Two independent clinical studies have suggested cut-off values of less than $350 \mathrm{fpm} \mathrm{[12]} \mathrm{and}$ $355 \mathrm{fpm}$ [13] for predicting spontaneous cardioversion. As all AF-induced horses receiving saline in this study, had AFR below this threshold and cardioverted spontaneously, it seems that these cut-off values may also apply to horses. Atrial remodelling will advance as AF accelerates as a consequence of AF itself. [14] As AFR can be used to assess atrial remodelling, it is reasonable to expect that AFR will evolve with increased AF duration. In agreement, we found the three horses with spontaneous persistent AF to have a mean of the first AFR value recorded at rest of $364 \pm 26$, which is considerably higher
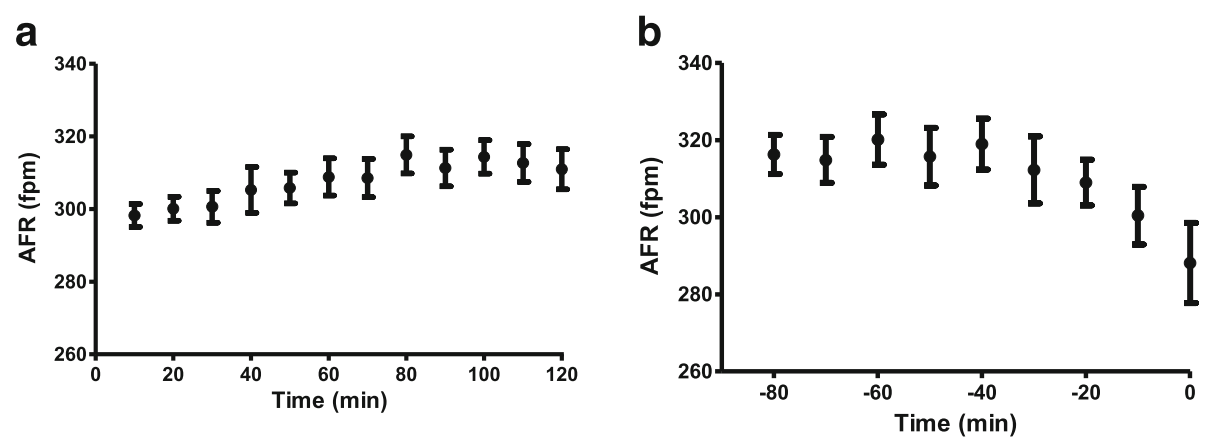

Fig. 1 AFR in control horses at AF onset (a) and before spontaneous cardioversion (b). a illustrates an increase in AFR in the first 70 min before reaching a plateau. $\mathbf{b}$ illustrates the gradual decrease in AFR 30 min before restoration of sinus rhythm at time $=0$. Values are presented as mean $\pm \mathrm{SEM}, n=7$ 

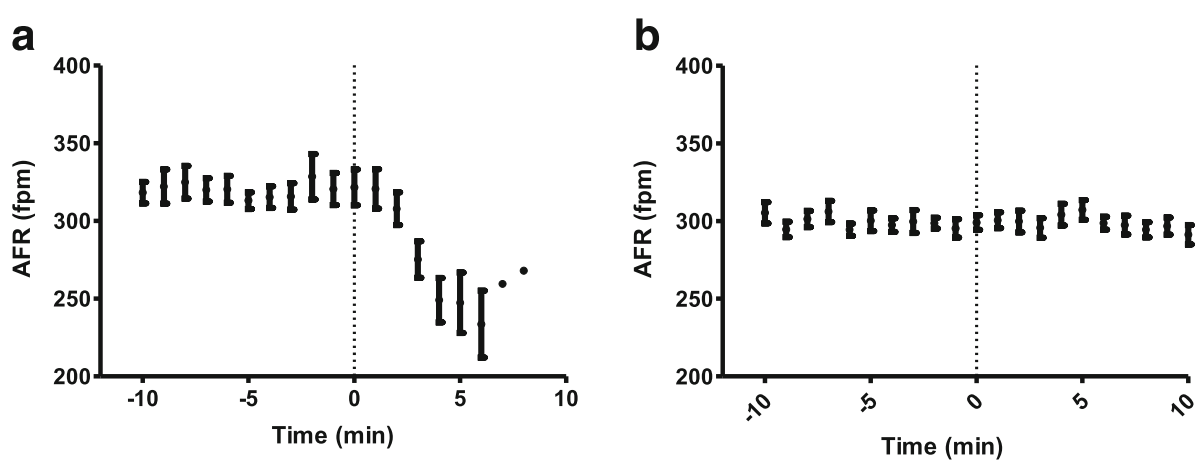

Fig. 2 AFR 10 min before and after treatment was initiated (at time $=0$ ). Data are presented as mean \pm SEM. a represents the horses treated with flecainide. Note the rapid change in AFR as a consequence of flecainide administration. $\mathbf{b}$ represents the horses treated with saline

than the short-term induced AF and also in agreement with human AF studies, in which patients with AF episodes longer than 3 months had a frequency of $402 \pm 78$ beats/min. [2]

\section{AFR dynamics in control horses}

In the present study, we found an increase in AFR from AF onset, and six out of seven horses had reached a plateau after $70 \mathrm{~min}$. Using similar methodology, Bollmann et al. [15] and Petrutiu et al. [16] reported time to a plateau being 4-5 min in patients with short AF episodes that lasted several minutes. Platonov et al. [11] observed an increase in AFR during the first 3 to $4 \mathrm{~h}$ in a study where one patient had $8.5 \mathrm{~h}$ of $\mathrm{AF}$, and the remaining three patients had $\mathrm{AF}$ episodes of more than 48 h. The mean AF duration in our control horses was $3.4 \mathrm{~h}$, suggesting that time to reach a plateau may depend on the duration of the AF episode, since both the length of the episodes and AFR are likely to reflect the degree of the atrial remodelling. This is further supported by the observations that no change in AFR was found when the duration of AF episodes was less than 15 min. [15] This study also indicates that horses with longer AF duration reached the plateau later. However, it is important to note that AF was induced in these horses, and that the sample size was rather small.

Before termination of AF, we observed a pronounced decrease in AFR. As with the initial AFR increase, the literature presents some inconsistencies on this matter Bollmann et al. [2] observed no changes in AFR towards termination using a 10-s time frame, while Petrutiu et al. [16] observed an abrupt decrease when AFR was studied on a second-to-second basis. In contrast, Platonov and colleagues [11] observed a gradual decrease $1 \mathrm{~h}$ before spontaneous cardioversion in one patient, while Fujiki [17] demonstrated a slowing of AFR 10 min prior to termination in patients that cardioverted in the morning. It is most likely that the different observations were due to different AF durations, suggesting that short-term AF episodes terminate more quickly with little or no change in AFR, while longer AF episodes have a gradual AFR decrease before spontaneous termination.

\section{The effect of flecainide on AFR}

Flecainide is a class I antiarrhythmic drug that slows down atrial fibrillatory process in horses $[3,8]$ and in humans. [18] The present study showed a clear effect of flecainide on AFR retrieved from surface ECGs, as AFR
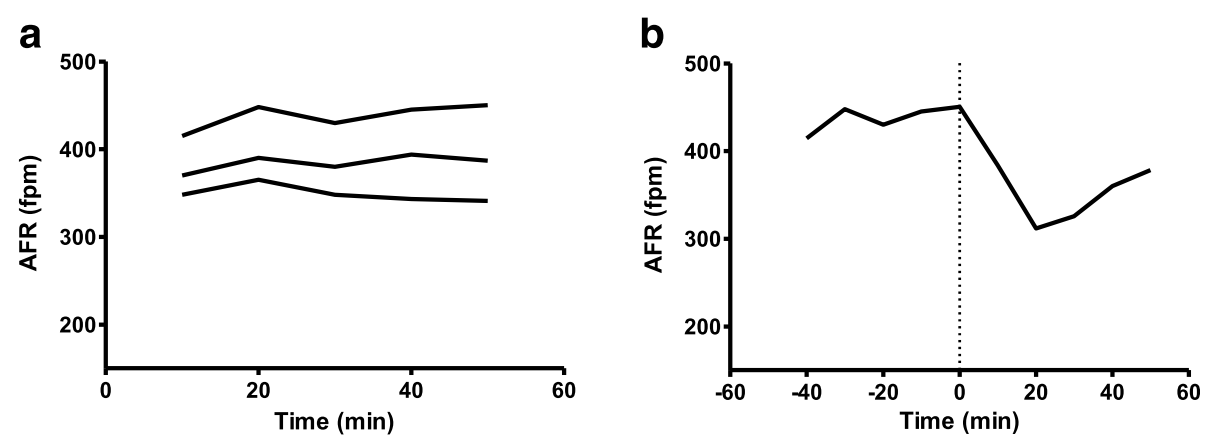

Fig. 3 AFR in horses with spontaneous persistent AF. Data are presented as 10-min average. a illustrates a stable AFR without drug intervention $(n=3)$ and $\mathbf{b}$ illustrates the attempt to terminate AF with flecainide (injection time $=0, n=1$ ). Note the significant decrease in AFR after flecainide infusion, followed by an increase as the flecainide failed to restore sinus rhythm 
dropped immediately after infusion started. The same effect was seen when 18 patients with AF were treated with oral flecainide, which resulted in a frequency drop of $30 \%$. [19] Patients with low frequency atrial fibrillation were more likely to cardiovert spontaneously or in response to treatment than patients with high frequency fibrillation. [15] Our study shows that this may also apply to horses, as flecainide terminated AF in the induced horses that had a significantly slower rate than those with spontaneous persistent AF. Flecainide reduced AFR by $100 \mathrm{fpm}$ to a level similar to the induced horses, yet this was not sufficient, as AFR started to increase again before cardioversion, leaving the horse in AF. Likewise, Bollmann et al. [15] showed that AF frequency decreases in response to flecainide treatment $(P<0.001)$ without restoring sinus rhythm. Several other studies have investigated the effect of a number of antiarrhythmic drugs, [20] all shown to reduce AFR. This supports AFR as a non-invasive method of tracking the effect of antiarrhythmic compounds in order to understand the effect on AF in humans and now also in horses.

\section{Conclusion}

To our knowledge, this is the first analysis of the noninvasive characterization of AF in horses. AFR values and their evolution during induced $\mathrm{AF}$ in horses resemble AFR dynamics in humans with paroxysmal AF, and horses with spontaneous persistent $\mathrm{AF}$ demonstrate an increase in AFR similar to patients with long-lasting AF. Flecainide caused a rapid decrease in AFR, suggesting that AFR measurement is a non-invasive method to monitor the effects of antiarrhythmic compounds in AF and supporting the validity of the horse as a model for human AF.

\section{Abbreviations}

AF: Atrial fibrillation; AFR: Atrial fibrillatory rate; BW: Body weight; ECG: Electrocardiogram; Fpm: Fibrillations per minute; IV: Intravenous

\section{Acknowledgements}

These studies were conducted at The University Hospital for Large Animals, Department of Veterinary Clinical Sciences, Faculty of Health and Medical Sciences, University of Copenhagen. We gratefully acknowledge all staff members for their support and the caretaking of the animals. Furthermore, we acknowledge Søren Saxmose Nielsen for invaluable and professional assistance with the statistical analyses. We also thank The Danish Council for Independent Research, The Danish Horse Levy Foundation and The Swedish Heart-Lung Foundation for financial support.

\section{Funding}

This work was supported by The Danish Council for Independent Research (DFF-1331-00313B), The Danish Horse Levy Foundation and The Swedish Heart-Lung Foundation (grants \#20140734 and \#20110875). The funding sources had no involvement in the conduct of the research or preparation of the article.

\section{Availability of data and materials}

The datasets used and/or analysed during the current study are available from the corresponding author on reasonable request.

\section{Authors' contributions}

Design and scientific rationale: EZH, MMH, HC, JC, SP, TJ, RB, PGP. Obtained funding: $M M H, H C, T J, P G P$, In vivo experiments: $M M H, H C$, EZH, analysis of ECG records: EZH, JC, PGP, Statistical analysis: EZH, HC, JC, Drafted manuscript: EZH, PGP, HC, RB, TJ, MMH, SP and JC. All authors read and approved the final manuscript.

\section{Ethics approval and consent to participate}

The studies were approved by The Danish Animal Experiments Inspectorate (license number 2012-15-2934-00198) and by the local ethical committee at University of Copenhagen. The horses induced with AF were retired trotters purchased as experimental animals whereas the horses with spontaneous AF was horses presented in the clinic at The University Hospital for Large Animals, Department of Veterinary Clinical Sciences, University of Copenhagen, due to AF. Owner consent was obtained for all horses participated in the study.

\section{Consent for publication}

Not applicable.

\section{Competing interests}

The authors declare that they have no competing interests.

\section{Publisher's Note}

Springer Nature remains neutral with regard to jurisdictional claims in published maps and institutional affiliations.

\section{Author details}

'Department of Veterinary Clinical Sciences, Faculty of Health and Medical Sciences, University of Copenhagen, Højbakkegaard Allé 5, 2630 Taastrup, Denmark. ${ }^{2}$ Department of Cardiology, Lund University, 21185 Lund, Sweden. ${ }^{3}$ Department of Cardiology, The Heart Centre, University of Copenhagen, Rigshospitalet, Blegdamsvej 9, 2100 Copenhagen, Denmark. ${ }^{4}$ Department of Biomedical Sciences, Faculty of Health and Medical Sciences, University of Copenhagen, Blegdamsvej 3, 2200 Copenhagen, Denmark. ${ }^{5}$ Arrhythmia Clinic, Skåne University Hospital and Department of Cardiology, Clinical Sciences, Lund University, 21185 Lund, Sweden.

Received: 15 June 2017 Accepted: 24 November 2017

Published online: 08 December 2017

\section{References}

1. Holm M, Pehrson S, Ingemansson M, Sornmo L, Johansson R, Sandhall L, Sunemark M, Smideberg B, Olsson C, Olsson SB. Non-invasive assessment of the atrial cycle length during atrial fibrillation in man: introducing, validating and illustrating a new ECG method. Cardiovasc Res. 1998;38(1):69-81.

2. Bollmann A, Kanuru NK, McTeague KK, Walter PF, DeLurgio DB, Langberg JJ. Frequency analysis of human atrial fibrillation using the surface electrocardiogram and its response to ibutilide. Am J Cardiol. 1998:81(12):1439-45.

3. Haugaard MM, Pehrson S, Carstensen H, Flethoj M, Hesselkilde EZ, Praestegaard KF, Diness JG, Grunnet M, Jespersen T, Buhl R. Antiarrhythmic and electrophysiologic effects of flecainide on acutely induced atrial fibrillation in healthy horses. J Vet Intern Med. 2015;29(1):339-47.

4. Haugaard MM, Hesselkilde EZ, Pehrson S, Carstensen H, Flethoj M, Praestegaard KF, Sorensen US, Diness JG, Grunnet M, Buhl R, et al. Pharmacologic inhibition of small-conductance calcium-activated potassium (SK) channels by NS8593 reveals atrial antiarrhythmic potential in horses. Heart Rhythm. 2015;12(4):825-35.

5. Else RW, Holmes JR. Pathological changes in atrial fibrillation in the horse. Equine Vet J. 1971;3(1):56-64.

6. Kirchhof P, Ammentorp B, Darius H, De Caterina R, Le Heuzey JY, Schilling RJ, Schmitt J, Zamorano JL. Management of atrial fibrillation in seven European countries after the publication of the 2010 ESC guidelines on atrial fibrillation: primary results of the PREvention of thromboemolic events-European registry in atrial fibrillation (PREFER in AF). Europace. 2014; 16(1):6-14.

7. Ohmura H, Nukada T, Mizuno Y, Yamaya Y, Nakayama T, Amada A. Safe and efficacious dosage of flecainide acetate for treating equine atrial fibrillation. J Vet Med Sci. 2000;62(7):711-5.

8. van Loon G, Blissitt KJ, Keen JA, Young LE. Use of intravenous flecainide in horses with naturally-occurring atrial fibrillation. Equine Vet $\mathrm{J}$. 2004;36(7):609-14 
9. De Clercq D, van Loon G, Tavernier R, Duchateau L, Deprez P. Atrial and ventricular electrical and contractile remodeling and reverse remodeling owing to short-term pacing-induced atrial fibrillation in horses. J Vet Intern Med. 2008:22(6):1353-9.

10. Stridh M, Sornmo L, Spatiotemporal QRST. Cancellation techniques for analysis of atrial fibrillation. IEEE Trans Biomed Eng. 2001;48(1):105-11.

11. Platonov PG, Stridh M, de Melis M, Urban L, Carlson J, Corbucci G, Holmqvist F. Analysis of atrial fibrillatory rate during spontaneous episodes of atrial fibrillation in humans using implantable loop recorder electrocardiogram. J Electrocardiol. 2012;45(6):723-6.

12. Choudhary MB, Holmqvist F, Carlson J, Nilsson HJ, Roijer A, Platonov PG. Low atrial fibrillatory rate is associated with spontaneous conversion of recent-onset atrial fibrillation. Europace. 2013;15(10):1445-52.

13. Husser D, Cannom DS, Bhandari AK, Stridh M, Sornmo L, Olsson SB, Bollmann A. Electrocardiographic characteristics of fibrillatory waves in new-onset atrial fibrillation. Europace. 2007;9(8):638-42.

14. Wijffels MC, Kirchhof CJ, Dorland R, Allessie MA. Atrial fibrillation begets atrial fibrillation. A study in awake chronically instrumented goats. Circulation. 1995;92(7):1954-68.

15. Bollmann A, Sonne K, Esperer HD, Toepffer I, Langberg JJ, Klein HU. Noninvasive assessment of fibrillatory activity in patients with paroxysmal and persistent atrial fibrillation using the Holter ECG. Cardiovasc Res. 1999:44(1):60-6.

16. Petrutiu S, Sahakian AV, Swiryn S. Short-term dynamics in fibrillatory wave characteristics at the onset of paroxysmal atrial fibrillation in humans. J Electrocardiol. 2007:40(2):155-60.

17. Fujiki A, Sakabe M, Nishida K, Mizumaki K, Inoue H. Role of fibrillation cycle length in spontaneous and drug-induced termination of human atrial fibrillation. Circ J. 2003;67(5):391-5.

18. Biffi M, Boriani G, Bronzetti G, Capucci A, Branzi A, Magnani B. Electrophysiologica effects of flecainide and propafenone on atrial fibrillation cycle and relation with arrhythmia termination. Heart. 1999;82(2):176-82.

19. Bollmann A, Binias KH, Toepffer I, Molling J, Geller C, Klein HU. Importance of left atrial diameter and atrial fibrillatory frequency for conversion of persistent atrial fibrillation with oral flecainide. Am J Cardiol. 2002;90(9):1011-4.

20. Platonov PG, Corino VD, Seifert M, Holmqvist F, Sornmo L. Atrial fibrillatory rate in the clinical context: natural course and prediction of intervention outcome. Europace. 2014;16(Suppl 4) iv110-iv119

\section{Submit your next manuscript to BioMed Central and we will help you at every step:}

- We accept pre-submission inquiries

- Our selector tool helps you to find the most relevant journal

- We provide round the clock customer support

- Convenient online submission

- Thorough peer review

- Inclusion in PubMed and all major indexing services

- Maximum visibility for your research

Submit your manuscript at www.biomedcentral.com/submit 